\title{
Expanding transcatheter aortic valve replacement into uncharted indications
}

\author{
Guson Kang and Juyong Brian Kim
}

Division of Cardiovascular Medicine, Department of Medicine and Cardiovascular Institute, Stanford University, Stanford, CA, USA

Received: January 9, 2018

Accepted: January 31, 2018

\section{Correspondence to}

Juyong Brian Kim, M.D.

Division of Cardiovascular Medicine, Department of Medicine and Cardiovascular Institute, Stanford University, 300 Pasteur Dr Rm CV273, Falk Bldg CVRC MC 5406, Stanford, CA 94305 , USA

Tel: $+1-650-725-2621$

Fax: +1-650-725-6766

E-mail: kimjb@stanford.edu
Since the first-in-man transcatheter delivery of an aortic valve prosthesis in 2002, the landscape of aortic stenosis therapeutics has shifted dramatically. While initially restricted to non-surgical cases, progressive advances in transcatheter aortic valve replacement and our understanding of its safety and efficacy have expanded its use in intermediate and possibly low surgical risk patients. In this review, we explore the past, present, and future of transcatheter aortic valve replacement.

Keywords: Transcatheter aortic valve replacement; Indications; Aortic valve stenosis

\section{INTRODUCTION}

Since the first description of its natural history by Ross and Braunwald [1] in 1968, aortic stenosis (AS) has gained recognition as a highly prevalent and morbid disease [2]. Recent cohort studies suggest that AS is present in nearly $4 \%$ of septuagenarians and in nearly $10 \%$ of octogenarians [3]. When symptomatic, AS carries a prognosis comparable with those of many end-stage cancers, with a 1-year mortality rate of $30 \%$ to $50 \%$ [4]. Since it was first performed by Harken in 1960, surgical valve replacement has been the only default therapy for severe AS for decades [5].

Transcatheter delivery was not achieved until 2002, when Cribier et al. [6] performed the first-in-man transcatheter aortic valve replacement (TAVR). Since then, TAVR has exploded into near global ubiquity. At least seven different devices are now commercially available. Furthermore, as the indications for TAVR continue to expand, procedural volumes have ballooned in step, more than doubling from 32,000 procedures in 2012 to over 70,000 in 2015 worldwide. By some estimates, TAVR is expected to overtake coronary stenting volumes by 2025 , with an anticipated procedural volume of 289,000 [7].

Despite its rapid growth, much about the clinical use of TAVR remains unknown [8]. Now that it is approved for commercial use in intermediate surgical risk patients, has TAVR reached the boundaries of its indicated use? Herein, we describe the past, present, and future of TAVR indications. 


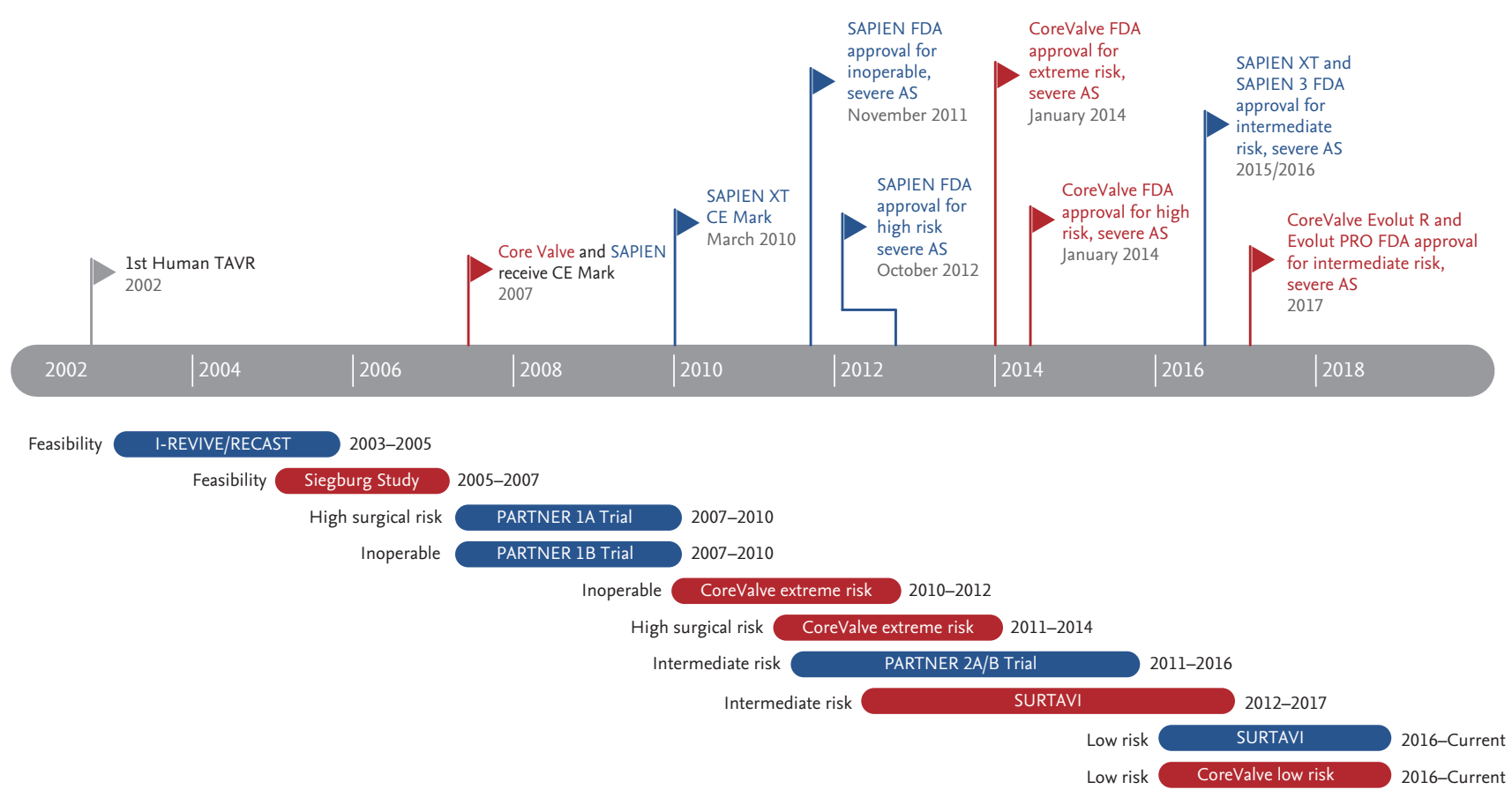

Figure 1. Timeline of major transcatheter aortic valve replacement (TAVR) trials and approvals. Events in red correspond to CoreValve; blue denotes SAPIEN. CE, Conformité Européenne; FDA, Food and Drug Administration; AS, aortic stenosis; I-REVIVE, Initial Registry of EndoVascular Implantation of Valves in Europe; RECAST, Registry of Endovascular Critical Aortic Stenosis Treatment; PARTNER, Placement of AoRTic traNscathetER valves; SURTAVI, Surgical or Transcatheter Aortic Valve Replacement in Intermediate-Risk Patients.

\section{THE PAST}

\section{Non-operable, severe, symptomatic AS}

Before the advent of TAVR, nearly one-thirds of patients with severe symptomatic AS were left untreated because they were not candidates for surgery [9]. After more than two decades without any progress, balloon aortic valvuloplasty (BAV) was introduced in 1985 [10]. Unfortunately, the potential of BAV to introduce aortic insufficiency has limited its clinical utility. More importantly, its long-term durability proved poor, and most valves were restenosed by 1 year.

The earliest TAVRs were performed exclusively on a compassionate-use basis in those with a prohibitively high surgical risk. The first two devices to be evaluated in humans were the balloon-expandable SAPIEN valve (Edwards Lifesciences, Irvine, CA, USA) and the self-expanding CoreValve (Medtronic, Minneapolis, MN, USA). The initial feasibility studies (I-REVIVE [Initial Registry of EndoVascular Implantation of Valves in Europe], RECAST [Registry of Endovascular Critical Aortic Stenosis Treatment], TRAVERCE [The Initial Multicenter
Feasibility Trial for TA-AVI], and REVIVAL [Transcatheter Endovascular Implantation of Valves study] for the SAPIEN valve [11]; the Siegburg first-in-man study for the CoreValve [12]) included only patients who had been rejected for surgery due to extreme comorbidities or contraindications. While these trials were plagued with high rates of early major adverse events (over 30\% in some cohorts), they demonstrated hemodynamic and symptomatic benefits [11]. In light of these data, both the SAPIEN transfemoral system and CoreValve earned their Conformité Européenne (CE) mark certifications for commercial sale in Europe in 2007 (Fig. 1), although the Food and Drug Administration (FDA) did not approve their use in the United States until after the first major clinical trials.

These first-in-man and feasibility studies paved the way for the first major clinical trials. Edwards Lifesciences received permission from the FDA to conduct the PARTNER (Placement of AoRTic traNscathetER valves) trial to evaluate the SAPIEN transcatheter system in March 2007. This landmark multi-arm trial, which compared TAVR with surgical therapy (Cohort A) 
or medical therapy (Cohort B), was the first large-scale multicenter randomized controlled trial to examine TAVR outcomes in high-risk or non-operable patients compared with surgical or medical therapy, respectively. Enrollment continued through 2009, and the initial results from the non-surgical arm (PARTNER 1B) were published in September 2010.

PARTNER 1B randomized 358 non-operable patients with severe symptomatic AS to receive the balloon-expandable SAPIEN valve or medical therapy (with or without BAV) and demonstrated an impressive $45 \%$ relative risk reduction in all-cause mortality at 1 year $30.7 \%$ after TAVR vs. 50.7\% after medical therapy) and a $54 \%$ relative risk reduction in combined major adverse cardiovascular and cerebrovascular events [4]. Patients were deemed non-operable if their predicted 30-day operative risk of morbidity or mortality exceeded $50 \%$. It was noted that the 30-day incidence of major stroke trended higher (5.0\% vs. $1.1 \%$ ) in the TAVR group. Despite this, PARTNER $1 B$ immediately established TAVR as the new standard of care for non-operable severe AS.

Given the clear inferiority of medical therapy alone in this patient population, similar randomized comparisons could not be made for self-expanding prostheses. Therefore, the subsequent CoreValve Extreme Risk United States study was performed as a single-arm trial that used the PARTNER $1 B$ data to set a performance goal for a 1-year mortality or stroke rate of $43 \%$. That trial, which examined 489 extreme-risk or non-operable patients (defined as a 30-day operative risk of morbidity or mortality $\geq 50 \%$ ), far exceeded these goals, with a combined mortality and major stroke rate of $26 \%$ at 1 year.

Approval in the United States has been slow despite two major trials demonstrating the benefits of both balloon-expandable and self-expanding prostheses in high-risk, non-operable patients with severe symptomatic AS over medical therapy. It took until November 2011 for the FDA to approve the SAPIEN transfemoral system: "The Edwards SAPIEN transcatheter heart valve sizes $23 \mathrm{~mm}$ and $26 \mathrm{~mm}$ and RetroFlex 3 Delivery System are indicated for transfemoral delivery in patients with severe, symptomatic, native-valve AS who have been determined by a cardiac surgeon to be inoperable for surgical aortic valve replacement (SAVR) and in whom existing comorbidities would not preclude the expected benefit from correction of the AS [13]." The panel also expressed concern regarding the elevated stroke risk seen in PARTNER $1 B$ and requested two post-approval studies, both to follow long-term outcomes and to observe neurological/vascular adverse outcomes and valve durability. Approval for the CoreValve system lagged even further behind, earning its first FDA approval in January 2014 for use in the same "extreme surgical risk" patients evaluated in the CoreValve Extreme Risk trial [14].

\section{High-risk, severe, symptomatic AS}

In parallel with the PARTNER $1 B$ trial, PARTNER $1 A$ randomized 699 patients with severe symptomatic AS and at high surgical risk to either the traditional open surgery group or the transfemoral or transapical TAVR group (SAPIEN) [15]. In this cohort, high risk was defined by a Society for Thoracic Surgeons (STS) risk of at least $10 \%$. TAVR was found to be non-inferior with respect to all-cause mortality at 1 year $(24.2 \%$ vs. $26.8 \%$ in the surgical group). Consistent with data from prior trials, early mortality was higher using the transapical approach than transfemoral delivery. Further subgroup analyses suggested that women and patients with a prior history of coronary artery bypass grafting benefited the most from transcatheter replacement.

The initial response to PARTNER 1A was one of cautious enthusiasm, tempered in large part by concerns over neurologic complications. Similar to the findings from PARTNER $1 B$, the risk of stroke was elevated in the PARTNER IA TAVR cohort. While not sufficiently powered to explore this endpoint in isolation, combined stroke or transient ischemic attack (TIA) was significantly more frequent with TAVR compared to surgery compared to surgery both at 30 days (19 patients vs. 8 patients, $p=0.04$ ) and at 1 year (27 patients vs. 13 patients, $p=0.04$ ), and TAVR showed a higher tendency of major stroke at 1 year ( 17 patients vs. 8 patients, $p=0.07$ ).

However, longer-term data have provided different insights. While mortality between the cohorts remained unchanged, the combined stroke/TIA risk converged with the passage of time: at 2 years, the risk was borderline significant (11.2\% vs. $6.5 \%, p=0.05$ ) [16], and at 5 years the risk was equivalent between the groups $(15.9 \%$ vs. $14.7 \%, p=0.35)[17]$. While TAVR potentially has an early or periprocedural risk of stroke, this may not prove 
relevant in the long-term.

The United States CoreValve High Risk Study was the second landmark study to examine TAVR in high (but not a prohibitive) surgical risk patients with severe AS and the first to examine self-expanding prostheses [18]. Similar to PARTNER, 795 patients were randomized to the SAVR or TAVR group. Interestingly, the results suggested superiority of TAVR over surgery in high surgical risk patients, with a statistically lower 1-year mortality rate (14.2\% vs. $19.1 \%, p=0.04$ for superiority). Furthermore, the short-term stroke rate was comparable between the TAVR and surgical groups (4.9\% vs. $6.2 \%$, respectively; $p=0.46$ ).

However, several key differences should be noted. In contrast to PARTNER, high risk was defined as a modified STS score corresponding to a short-term mortality rate of greater than 15\%, which was higher than the $10 \%$ cutoff rate used in PARTNER. Furthermore, the CoreValve High Risk Study did not assess transapical access (although it did include direct aortic or subclavian access), whereas 104 of 348 patients in PARTNER 1A underwent transapical TAVR. While an initial case report did suggest that transapical delivery using a CoreValve device was possible [19], it has since been labeled only for transvascular (femoral, subclavian, or direct aortic) delivery [20].

Regardless of the brand of prosthesis, these studies expanded the indications for TAVR to include high surgical risk patients. The FDA subsequently approved the SAPIEN and CoreValve systems for use in high surgical risk patients with symptomatic severe AS in October 2012 and June 2014, respectively [21,22].

\section{THE PRESENT}

\section{Intermediate-risk, severe, symptomatic AS}

The latest step in expanding TAVR indications was the inclusion of patients with an "intermediate surgical risk" as candidates.

Preceded by several smaller trials, PARTNER 2 (2016) has been the largest such trial [23-25]. The trial primarily evaluated Edwards' second-generation balloon-expandable device, the SAPIEN XT, which was marketed to have thinner struts, more sizing options, and a lower profile delivery system. PARTNER 2 also included co- horts to examine the SAPIEN XT in inoperable (Cohort B) patients, as well as the third-generation SAPIEN 3 in all three risk strata (inoperable, high surgical risk, and intermediate surgical risk). In Cohort A, 2,032 patients who met the entry criteria for "intermediate surgical risk," defined as a Society of Thoracic Surgeons Predicted Risk of Mortality (STS-PROM) of $4 \%$ to $8 \%$, were randomized to the TAVR or surgery group. TAVR again proved non-inferior to conventional surgery with respect to the primary endpoints of mortality and disabling stroke (TAVR vs. surgery: 19.3\% vs. $21.1 \%$; hazard ratio, $0.79 ; 95 \%$ confidence interval, 0.62 to 1.00). Two other findings from this trial are important to note: (1) the stroke/TIA rates did not differ between the groups during the 2-year follow-up period, and (2) transfemoral TAVR was superior to surgery with respect to the primary endpoint, demonstrating the potential for TAVR not only as an alternative to surgery but also as the preferred treatment modality.

Medtronic responded in suit, presenting the Surgical or Transcatheter Aortic Valve Replacement in Intermediate-Risk Patients (SURTAVI) trial the following year [26]. The trial evaluated primarily the original CoreValve prosthesis, but also the second iteration (Evolut R) in 16\% of the TAVR group. Similar to the high surgical risk trials, SURTAVI employed a different threshold criterion for intermediate risk than that of PARTNER 2A, allowing an STS-PROM range of $3 \%$ to $15 \%$. This trial confirmed the findings from PARTNER $2 \mathrm{~A}$ and found that TAVR was non-inferior to surgery with respect to the primary endpoint of death or disabling stroke at 2 years.

These trials launched a battery of recent FDA approvals: the SAPIEN XT was approved for high surgical risk and inoperable patients (2015) [27], pulmonic procedures (2016) [28], and intermediate surgical risk patients (2016) [29]; the SAPIEN 3 was approved in parallel for high surgical risk and inoperable patients (2015) [30] and intermediate surgical risk patients (2016) [29]; and the CoreValve, Evolut R, and third-generation Evolut PRO were approved for intermediate surgical risk patients (2017) [31].

\section{THE FUTURE}

\section{Low-risk, severe, symptomatic AS}

Of patients with severe AS, 79.9\% with severe AS are clas- 
sified as low-risk (STS database, 2002 to 2010). Therefore, expanding the commercial availability of TAVR to include this subpopulation could effectively quadruple the eligible patient population. In the background of this enormous potential, the first randomized trial to examine low-risk patients was the small Nordic Aortic Valve Intervention (NOTION) trial, which randomized 280 low surgical risk $(\mathrm{STS}<4 \%$ ) patients to receive first-generation CoreValve TAVR or surgery [24]. While clearly underpowered, the results were promising: no significant difference in the major adverse cardiovascular or cerebrovascular events (primary endpoint) was observed at 1 year (13.1\% vs. 16.3\%, $p=0.43$ ).

Valve manufacturers have since initiated two significantly larger trials to evaluate the low surgical risk population. The Medtronic TAVR in low-risk patients trial (NCTo2701283) began in March 2016 with a projected enrollment of 1,200 low surgical risk patients. The protocol defines low risk as a predicted risk of 30-day mortality from surgery of less than $3 \%$ and randomizes patients to TAVR using the original CoreValve or CoreValve Evolut R prosthesis group or surgery group. Follow-up to evaluate the combined primary endpoint of all-cause mortality and stroke at 2 years is expected to be complete in March 2018.

PARTNER 3 (NCTo2675114) began enrolling patients in April 2016 with severe calcific AS and a low surgical risk, defined as an STS score of less than $4 \%$, and it is anticipated to enroll 1,328 patients through October 2018. Participants are being randomized to the transfemoral TAVR using the SAPIEN 3 prosthesis or surgical bioprosthetic AVR group. The investigators will examine the primary composite outcome of all-cause mortality, stroke, and re-hospitalization at 1 year over an extended follow-up period of 10 years. As the surgical risk profiles lower, we anticipate the proportion of younger patients to increase in step, and given that young age is not an exclusion criterion for PARTNER 3, this raises important questions regarding the long-term durability of the TAVR bioprostheses.

\section{Severe asymptomatic AS}

Every TAVR trial thus far has compared TAVR with surgery in symptomatic patients, primarily based off natural history data suggesting that severe AS accompanied by symptoms heralds a sudden decrease in survival.
What about the severe hemodynamically asymptomatic patients?

Here, we turn to the surgical literature for insight. While surgery is still uncommon in asymptomatic patients, it has been explored in certain subpopulations. Observational studies have suggested that all-cause mortality benefits may be seen in asymptomatic patients with very severe or critical AS, usually defined as an aortic valve area (AVA) $\leq 0.75 \mathrm{~cm}^{2}$ and a peak velocity $\geq$ $4.5 \mathrm{~m} / \mathrm{sec}$, or with a mean gradient $\geq 50 \mathrm{mmHg}$ [32]. Other studies have suggested benefits of SAVR in patients with concomitant left ventricular dysfunction, currently a $1 \mathrm{~B}$ indication per the 2014 American College of Cardiology/American Heart Association (ACC/AHA) guidelines for the management of patients with valvular heart disease [33,34]. More recent observational data from the CURRENT-AS (Contemporary outcomes after sURgery and medical tREatmeNT in patients with severe Aortic Stenosis) registry in Japan also suggest better mortality and heart failure hospitalization outcomes during early surgery in severe AS patients without symptoms [35].

EARLY-TAVR (Evaluation of Transcatheter Aortic Valve Replacement Compared to SurveilLance for Patients With AsYmptomatic Severe Aortic Stenosis) (NCTo3042104) is a large, prospective, randomized controlled trial that began enrolling asymptomatic patients with severe AS in July 2017, randomizing to either the TAVR using the SAPIEN 3 prosthesis or surveillance alone group. A slower enrollment cadence is anticipated, with a goal of 1,109 patients and completion of the primary endpoint follow-up by December 2021. A positive trial of severe asymptomatic AS would have significant implications for current echocardiographic screening guidelines.

\section{Low-gradient, severe AS}

The major prospective TAVR trials have generally required hemodynamic evidence of stenosis severity, using an entry criterion of a peak transvalvular velocity $\geq$ $4 \mathrm{~m} / \mathrm{sec}$ or a mean transvalvular gradient $\geq 40 \mathrm{mmHg}$ to define "severe" AS. There are, however, subgroups of patients with extremely small AVAs and lower gradients, or "low-gradient, severe AS" (LGAS), who may benefit from valvular replacement but do not meet the classical hemodynamic criteria. LGAS has generally been defined as having a mean pressure gradient $\leq 40 \mathrm{mmHg}$ and AVA $\leq$ 
$1 \mathrm{~cm}^{2}$, but it can be further subdivided into low flow/low gradient with reduced ejection fraction $(\mathrm{EF}<50 \%)$, low flow/low gradient with preserved EF, or normal flow/ low gradient. The accuracy of the various AVA equations depends on cardiac output to variable degrees (e.g., the Gorlin vs. continuity equations), complicating the distinction between truly severe LGAS and "pseudo-severe" LGAS. The best way to approach these patients, both from classification and management standpoints, remains unclear. Current guidelines list aortic valve replacement as a class IIA recommendation for patients with symptomatic, low-flow LGAS with a reduced EF if low-dose dobutamine echocardiography demonstrates an augmentation of mean aortic valve gradient to $\geq 40$ mmHg, but evidence is lacking [34].

Although the concept of "LGAS" was recognized early on [36], the burgeoning of transcatheter valvular replacement has resulted in closer scrutiny of these clinical subgroups. TAVR UNLOAD (Transcatheter Aortic Valve Replacement to UNload the Left Ventricle in Patients With ADvanced Heart Failure) (NCTo2661451) is one such current trial evaluating the efficacy of TAVR using the SAPIEN 3 prosthesis versus optimal medical therapy in patients with hemodynamically moderate AS (including patients with $\mathrm{AVA}<1.0 \mathrm{~cm}^{2}$ ) and an $\mathrm{EF}<50 \%$. Enrollment for the trial began in September 2016 and is expected to complete follow-up of an anticipated 600 patients through 2020 .

\section{Refining patient selection}

With the establishment of TAVR as a viable alternative to traditional SAVR in large clinical trials involving heterogeneous populations, the need for TAVR-specific risk stratification tools has emerged. As mentioned earlier, all major trials thus far have used surgical risk calculators (STS PROM and EuroSCORE) to estimate procedural risk in all AS patients. While these trials have provided insight into treatment at the population level, optimal management of individual patients ultimately requires balancing surgical risk using a TAVR-specific risk model.

In 2011, the STS/ACC Transcatheter Valve Therapy (T'VT) Registry was developed in part to address this need. The model was published in 2016, before which no such model had achieved the same statistical power or adoption [37]. The model included data from 13,718 patients in the United States and found that age, estimated glomerular filtration rate, need for hemodialysis, New York Heart Association functional class IV, severe chronic lung disease, need for non-femoral access site, and higher procedural acuity were associated with in-hospital TAVR mortality; the associated risk calculator is available online or as a mobile application [38]. New risk scores have since been proposed that incorporate different covariates (albumin, home oxygen use, or assisted living) [39], although the STS/ACC TVT Registry score remains the most widely used.

More recently, adjunctive modalities, such as biomarkers or imaging, have become active areas of investigation to refine traditional clinical risk models. While non-specific, several biomarkers associated with cardiovascular stress have been demonstrated to have prognostic value for TAVR. Growth differentiation factor 15 , soluble ST2, and N-terminal pro-brain natriuretic peptide have all been implicated as predictive biomarkers for periprocedural TAVR outcomes and long-term left ventricular remodeling, both in isolation and in combination with the STS/ACC TVT risk calculator $[40,41]$. Echocardiographic assessment of ventricular remodeling and recovery following TAVR may also help predict which patients will benefit most from TAVR and close follow-up [40,42]. Echocardiographic and computed tomography-guided measurements have also proved useful in predicting procedural outcomes, such as acute mitral regurgitation [43] and aortic regurgitation [44].

\section{CONCLUSIONS}

There have been exponential growth of transcatheter valvular therapies and their approved indications, with more exciting developments to follow. We anticipate that trials examining TAVR in low-risk patient groups (dubbed the surgeon's "sweet spot") will either shift the landscape in favor of transcatheter delivery or cement surgery's place in the arsenal of AS therapy. Indeed, it may even reach beyond the traditional scope of surgery, given the recent push towards patients with severe asymptomatic AS or LGAS. Data from these trials will eventually need to be interpreted in the setting of improved TAVR-specific risk models for best management of the individual patient. Finally, the landscape of TAVR 
research continues to expand, and many questions remain, especially regarding prosthesis durability. As the pivotal TAVR trials cross the decade mark, more longterm durability data will be available to resolve one of the largest remaining questions in the field — that is, until the next valve is released.

\section{Conflict of interest}

No potential conflict of interest relevant to this article was reported.

\section{REFERENCES}

1. Ross J Jr, Braunwald E. Aortic stenosis. Circulation 1968;38(1 Suppl):61-67.

2. Bonow RO, Greenland P. Population-wide trends in aortic stenosis incidence and outcomes. Circulation 2015;131:969-971.

3. Eveborn GW, Schirmer H, Heggelund G, Lunde P, Rasmussen K. The evolving epidemiology of valvular aortic stenosis. The Tromso study. Heart 2013;99:396-400.

4. Leon MB, Smith CR, Mack M, et al. Transcatheter aorticvalve implantation for aortic stenosis in patients who cannot undergo surgery. N Engl J Med 2010;363:1597-1607.

5. Harken DE, Taylor WJ, Lefemine AA, et al. Aortic valve replacement with a caged ball valve. Am J Cardiol 1962; 9:292-299.

6. Cribier A, Eltchaninoff H, Bash A, et al. Percutaneous transcatheter implantation of an aortic valve prosthesis for calcific aortic stenosis: first human case description. Circulation 2002;106:3006-3008.

7. Leon MB. Global TAVR market poised to exceed DES market by 2025 [Internet]. TCTMD, 2015 [cited 2018 Feb 7]. Available from: https://www.tctmd.com/news/global-tavrmarket-poised-exceed-des-market-2025.

8. Tastet L, Simard L, Clavel MA. Severe and asymptomatic aortic stenosis management challenge: knowing that we do not really know. Curr Treat Options Cardiovasc Med 2017;19:33.

9. Iung B, Baron $\mathrm{G}$, Butchart EG, et al. A prospective survey of patients with valvular heart disease in Europe: the Euro Heart Survey on Valvular Heart Disease. Eur Heart J 2003;24:1231-1243.

10. Cribier A, Savin T, Saoudi N, Rocha P, Berland J, Letac B. Percutaneous transluminal valvuloplasty of acquired aortic stenosis in elderly patients: an alternative to valve replacement? Lancet 1986;1:63-67.

11. Cribier A, Eltchaninoff H, Tron C, et al. Treatment of calcific aortic stenosis with the percutaneous heart valve: mid-term follow-up from the initial feasibility studies: the French experience. J Am Coll Cardiol 2006;47:12141223.

12. Grube E, Laborde JC, Gerckens U, et al. Percutaneous implantation of the CoreValve self-expanding valve prosthesis in high-risk patients with aortic valve disease: the Siegburg first-in-man study. Circulation 2006;114:1616-1624.

13. Barbash IM, Waksman R. Overview of the 2011 Food and Drug Administration Circulatory System devices panel of the Medical Devices Advisory Committee meeting on the Edwards SAPIEN ${ }^{\text {TM }}$ transcatheter heart valve. Circulation 2012;125:550-555.

14. Food and Drug Administration. Corevalve Extreme Risk approval letter [Internet]. Silver Spring (MD): FDA, 2014 [cited $2018 \mathrm{Feb} 7]$. Available from: https://www.accessdata. fda.gov/cdrh_docs/pdfi3/P130021a.pdf.

15. Smith CR, Leon MB, Mack MJ, et al. Transcatheter versus surgical aortic-valve replacement in high-risk patients. $\mathrm{N}$ Engl J Med 2011;364:2187-2198.

16. Kodali SK, Williams MR, Smith CR, et al. Two-year outcomes after transcatheter or surgical aortic-valve replacement. N Engl J Med 2012;366:1686-1695.

17. Mack MJ, Leon MB, Smith CR, et al. 5-Year outcomes of transcatheter aortic valve replacement or surgical aortic valve replacement for high surgical risk patients with aortic stenosis (PARTNER 1): a randomised controlled trial. Lancet 2015;385:2477-2484.

18. Adams DH, Popma JJ, Reardon MJ, et al. Transcatheter aortic-valve replacement with a self-expanding prosthesis. N Engl J Med 2014;370:1790-1798.

19. Lange R, Schreiber C, Gotz W, et al. First successful transapical aortic valve implantation with the Corevalve revalving system: a case report. Heart Surg Forum 2007;10:E478-E479.

20. Medtronic. CoreValve important safety information [Internet]. Luxembourg City (LU): Medtronic CV Luxembourg S.a.r.1., c2015 [cited 2018 Feb 7]. Available from: http://www.corevalve.com/us/safety-information/ index.htm.

21. Wood S. FDA expands Sapien TAVI indication to highrisk surgical patients [Internet]. New York (NY): Medscape 
Medical News, c1994 [cited $2018 \mathrm{Feb} 7$ ]. Available from: https://www.medscape.com/viewarticle/772966.

22. Medtronic. Medtronic CoreValve ${ }^{\circledR}$ system receives FDA approval for patients at high risk for surgery [Internet]. Minneapolis (MN): Medtronic, c2015 [cited $2018 \mathrm{Feb} 7$ ]. Available from: http://newsroom.medtronic.com/phoenix. zhtml?c=251324\&p=irol-newsArticle\&ID=1939539.

23. Nielsen $\mathrm{HH}$, Klaaborg KE, Nissen $\mathrm{H}$, et al. A prospective, randomised trial of transapical transcatheter aortic valve implantation vs. surgical aortic valve replacement in operable elderly patients with aortic stenosis: the STACCATO trial. EuroIntervention 2012;8:383-389.

24. Thyregod HG, Steinbruchel DA, Ihlemann N, et al. Transcatheter versus surgical aortic valve replacement in patients with severe aortic valve stenosis: 1-year results from the all-comers NOTION Randomized Clinical Trial. J Am Coll Cardiol 2015;65:2184-2194.

25. Leon MB, Smith CR, Mack MJ, et al. Transcatheter or surgical aortic-valve replacement in intermediate-risk patients. N Engl J Med 2016;374:1609-1620.

26. Reardon MJ, Van Mieghem NM, Popma JJ, et al. Surgical or transcatheter aortic-valve replacement in intermediaterisk patients. N Engl J Med 2017;376:1321-1331.

27. Food and Drug Administration. Edwards SAPIEN XT high risk approval letter [Internet]. Silver Spring (MD): FDA, 2015 [cited $2018 \mathrm{Feb}$ 7]. Available from: https://www. accessdata.fda.gov/cdrh_docs/pdfi3/P130009So34a.pdf.

28. Edwards Lifesciences. Edwards SAPIEN XT valve receives FDA approval for pulmonic procedures [Internet]. Irvine (CA): Edwards Lifesciences Corporation, c2018 [cited $2018 \mathrm{Feb} 7]$. Available from: http://www.edwards.com/ ns20160302.

29. Food and Drug Administration. FDA approves expanded indication for two transcatheter heart valves for patients at intermediate risk for death or complications associated with open-heart surgery [Internet]. Silver Spring (MD): FDA, 2016 [cited $2018 \mathrm{Feb}$ 7]. Available from: https://www. fda.gov/NewsEvents/Newsroom/PressAnnouncements/ ucm517281.htm.

30. Food and Drug Administration. Edwards SAPIEN 3 high risk approval letter [Internet]. Silver Spring (MD): FDA, 2015 [cited $2018 \mathrm{Feb}$ 7]. Available from: https://www. accessdata.fda.gov/cdrh_docs/pdfi4/P140031a.pdf.

31. Medtronic. Medtronic expands TAVR access to more patients with symptomatic, severe aortic stenosis upon intermediate risk FDA approval [Internet]. Minneapolis
(MN): Medtronic, c2015 [cited 2018 Feb 7]. Available from: http://newsroom.medtronic.com/phoenix. zhtml?c=251324\&p=irol-newsArticle\&ID=2285395.

32. Kang DH, Park SJ, Rim JH, et al. Early surgery versus conventional treatment in asymptomatic very severe aortic stenosis. Circulation 2010;121:1502-1509.

33. Connolly HM, Oh JK, Orszulak TA, et al. Aortic valve replacement for aortic stenosis with severe left ventricular dysfunction. Prognostic indicators. Circulation 1997; 95:2395-2400.

34. Nishimura RA, Otto CM, Bonow RO, et al. 2014 AHA/ ACC guideline for the management of patients with valvular heart disease: executive summary: a report of the American College of Cardiology/American Heart Association Task Force on Practice Guidelines. J Am Coll Cardiol 2014;63:2438-2488.

35. Taniguchi $\mathrm{T}$, Morimoto $\mathrm{T}$, Shiomi $\mathrm{H}$, et al. Initial surgical versus conservative strategies in patients with asymptomatic severe aortic stenosis. J Am Coll Cardiol 2015; 66:2827-2838.

36. Shively BK. Evolving concepts about aortic stenosis. Cardiol Rev 1998;6:325-333.

37. Edwards FH, Cohen DJ, O'Brien SM, et al. Development and validation of a risk prediction model for in-hospital mortality after transcatheter aortic valve replacement. JAMA Cardiol 2016;1:46-52.

38. American College of Cardiology; The Society of Thoracic Surgeons. TAVR in-hospital mortality risk calculator [Internet]. American College of Cardiology; The Society of Thoracic Surgeons, 2016 [cited 2018 Feb 7]. Available from: http:/tools.acc.org/tavrrisk/\#!/content/evaluate/.

39. Popma JJ, Adams DH, Reardon MJ, et al. Transcatheter aortic valve replacement using a self-expanding bioprosthesis in patients with severe aortic stenosis at extreme risk for surgery. J Am Coll Cardiol 2014;63:19721981.

40. Kim JB, Kobayashi Y, Moneghetti KJ, et al. GDF-15 (growth differentiation factor 15) is associated with lack of ventricular recovery and mortality after transcatheter aortic valve replacement. Circ Cardiovasc Interv 2017;10:e005594.

41. Lindman BR, Breyley JG, Schilling JD, et al. Prognostic utility of novel biomarkers of cardiovascular stress in patients with aortic stenosis undergoing valve replacement. Heart 2015;101:1382-1388.

42. Kobayashi Y, Kim JB, Moneghetti KJ, et al. Dynamic 
changes in aortic impedance after transcatheter aortic valve replacement and its impact on exploratory outcome. Int J Cardiovasc Imaging 2017;33:1693-1701.

43. Ito A, Iwata S, Mizutani K, et al. Echocardiographic parameters predicting acute hemodynamically significant mitral regurgitation during transfemoral transcatheter aortic valve replacement. Echocardiography 2018;35:353-
360.

44. Roule V, Placente A, Sabatier R, et al. Angles between the aortic root and the left ventricle assessed by MDCT are associated with the risk of aortic regurgitation after transcatheter aortic valve replacement. Heart Vessels 2018;33:58-65. 